\title{
Design of Multimedia Playback System Based on Computer Network and New Media Technology
}

\author{
Haoxue Hui \\ Information and Modern Education Technology Centre, Xi'an University, 710065
}

\begin{abstract}
Keywords: Computer Network; New Media Technology; Multimedia Player; Multimedia Playback System
\end{abstract}

\begin{abstract}
Multimedia playback systems based on computer networks and new media technologies are increasingly emerging, driving the rapid growth of new media on the Internet. The speed at which people receive knowledge is also accelerating with the development of multimedia computer network technology. Multimedia technology was first proposed by Point Cast Network of the United States. The multimedia playback system is essentially a technology that selects specific information from a database and presents the information to the user in a certain way according to certain technical standards and methods. Based on the research of computer network and new media technology, this paper explores the design of multimedia playback system for computer network and new media technology.
\end{abstract}

\section{The Design Theory of Multimedia Playback System}

There are many types of multimedia playback systems. In terms of equipment, there are more application systems than the original computer network models, and there is no unified operating system. The application field of multimedia playback system has been involved in many industries, and the geographical scope is wide and the location is not concentrated. According to the above situation, when designing the multimedia playback system based on computer network and new media technology, we not only meet the actual needs but also make overall planning, considering the whole and paying attention to details. Based on actual conditions and needs, we have established the goals and design principles for the design of multimedia playback systems based on computer networks and new media technologies.

A general multimedia system consists of four parts, including a multimedia hardware, a multimedia system, a media processing tool, and a user application software. The design of the multimedia playback system generally has the following features. It has process control capability and can provide a guiding framework for the elements, and the media used in the multimedia playback system of computer network and new media technology include texts, pictures, sounds and movies, and various interactive function. Besides, it support for many hard devices and file formats to generate complex products with many media elements. Although multimedia data includes multiple tools for multiple media editing functions, its production capabilities are not as good as professional software. In addition, it is easy to get started but not easy to master, and it is no need for professional programmers to have programming experience. Achieve certain specific basic requirements through proficient use. However, it is inconvenient to modify content that is often modified and has high timeliness requirements. The main functions of each module of multimedia playback system based on computer network and new media technology are as following. According to the user's design requirements, it can provide an intuitive, efficient and convenient editing method for graphics, text, sound and image data integration and edit and integrate various media data.

The goals that the multimedia playback system should achieve are as follows. It can achieve resource sharing, and operate multi-user software. It is easy to operate, manage and maintain network. Besides, it can be interconnected with Internet and has good scalability. It can be used in all current network environments and hardware environments because of its good compatibility.

The design principles of multimedia playback systems based on computer networks and new media technologies are as follows. (1) Advancement and maturity: The network should adopt 
advanced and mature technologies. (2) Openness and standardness: In order to gradually develop and expand the multimedia playback system, it is necessary to adapt to different usage environments. (3) Effectiveness and sharing: The multimedia playback system should make full use of the user's existing equipment and resources, and the technology should continue to develop with the needs of users. (4) Security and reliability: The multimedia playback system should be safe and reliable during operation. (5) Operability and ease of use: The multimedia playback system must have certain operability, and the work of the system use and maintenance personnel is easy to use. The method overcomes the weakness of the existing authoring tools and is limited by the existing system, and combines the advantages of descriptive language and iconic creation tools. Using a combination of system architecture integration and computer programming technology, the existing powerful multimedia processing tools are used to shorten the development cycle to create the best multimedia playback system.

\section{The Multimedia Player Architecture}

The general multimedia player architecture design is shown in Figure 2.1. Located in the linux user space, the embedded linux system is supported by pure $\mathrm{C}+$ language in order to ensure the portability of the multimedia playback system. The processing of file input combined with MIME is mainly to read and parse the file content such as the length of the file specified by the user, the audio and video frame buffer, the encoding format, the playing time, and the file title, and display the pre-designed window. The plugin interface call is mainly to find the corresponding decoder plugin according to the file information area parsed in the previous part, and integrate all the operations of the decoder into a unified open interface and call. Through the class library provided by the plugin interface call, according to the need to design a basic window related to the management multimedia file that can directly interact with the user to open, delete, display files and play, and the window for managing and controlling the playback set by the user are designed. By implementing the core-interface call of the multimedia player, the scalability of the player can be greatly improved. The advantages of this pure $\mathrm{C}$ language development multimedia playback system are stable and compatible. The disadvantage is that pure $\mathrm{C}$ language development is difficult to read, and hardware acceleration is slightly obstacle. 


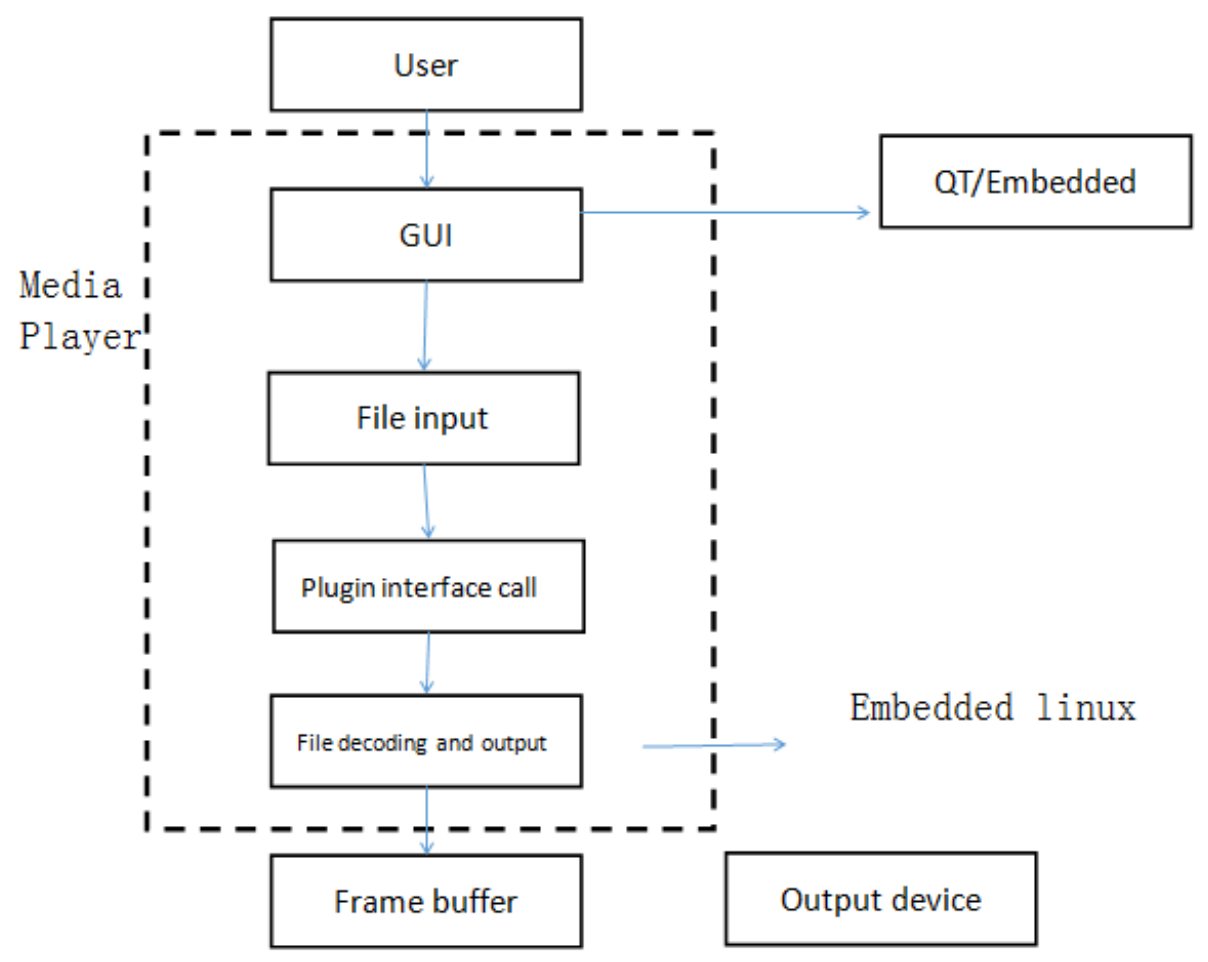

Figure 2.1. Embedded Multimedia Player Architecture Diagram

\section{The Functional Requirements of Multimedia Playback System}

A multimedia playback system refers to a system that processes and controls multimedia information using computer networks and new media technologies. The form in Table 3.1 is given according to the functional division of the multimedia playback system and gives the internal interface requirements specifications.

Table 3.1

\begin{tabular}{|c|c|c|}
\hline No. & $\begin{array}{l}\text { Functional } \\
\text { description }\end{array}$ & interface requirements \\
\hline 1. & File read-in & Input \\
\hline 2. & Format recognition & Treating processes \\
\hline 3. & $\begin{array}{l}\text { Decoding the } \\
\text { transcoding }\end{array}$ & $\begin{array}{lll}\text { (3) } & \text { Output } & \\
\text { (4) } & \text { Interface interaction }\end{array}$ \\
\hline 4. & play & process \\
\hline 5. & $\begin{array}{l}\text { Play progress } \\
\text { adjustment }\end{array}$ & $\begin{array}{l}\text { (5) Message and } \\
\text { communication }\end{array}$ \\
\hline 6. & View processing & protocol \\
\hline 7. & screenshot & Performance \\
\hline 8. & Users login & requirements \\
\hline 9. & $\begin{array}{l}\text { Account } \\
\text { management }\end{array}$ & $\begin{array}{ll}\text { (7) } & \text { Fault handling } \\
\text { (8) } & \text { Other requirements }\end{array}$ \\
\hline
\end{tabular}

The hierarchy of the multimedia system is identical in principle to the structure of the computer system. The function of the multimedia playback system is mainly manifested in the fact that the processing of sound video is composed of a hardware system and a software system and is played by using a computer language audio and video file. The hierarchical structure of the multimedia system only considers the characteristics of multimedia, and the content of each level is different. 
Table 3.2

\begin{tabular}{|c|l|}
\hline $\begin{array}{c}\text { Media } \\
\text { decoding }\end{array}$ & $\begin{array}{l}\text { Can identify and open current mainstream audio and video files } \\
\text { such as *.mid, *.mp3, *.wav, *.wma, *.wmv, *.AVI, *.rmvb, etc. } \\
\text { and animation culture such as *.fla, *.flv, *.swf, etc. }\end{array}$ \\
\hline Play control & $\begin{array}{l}\text { Play, pause, stop, previous, next, and other basic playback controls } \\
\text { for open multimedia files. The media playback progress can be } \\
\text { changed by dragging and clicking the drag bar, and the volume } \\
\text { control such as the volume increase effect and the mute can be } \\
\text { performed. }\end{array}$ \\
\hline $\begin{array}{c}\text { Auxiliary } \\
\text { playback } \\
\text { function }\end{array}$ & $\begin{array}{l}\text { Full-screen playback, playback for display, window scaling, } \\
\text { screenshots of video windows, click-to-play of playlists, and } \\
\text { emptying of databases. }\end{array}$ \\
\hline $\begin{array}{c}\text { Interface and } \\
\text { exception } \\
\text { handling }\end{array}$ & $\begin{array}{l}\text { The interface is simple and friendly. The operation is simple, and } \\
\text { the user's misoperation can be handled, which can reduce the } \\
\text { occurrence of abnormal program crash. }\end{array}$ \\
\hline
\end{tabular}

The multimedia playback system is an information technology that combines texts, graphics, images, animation, and sound through a computer, and performs integrated processing and control to complete a series of interactive operations.

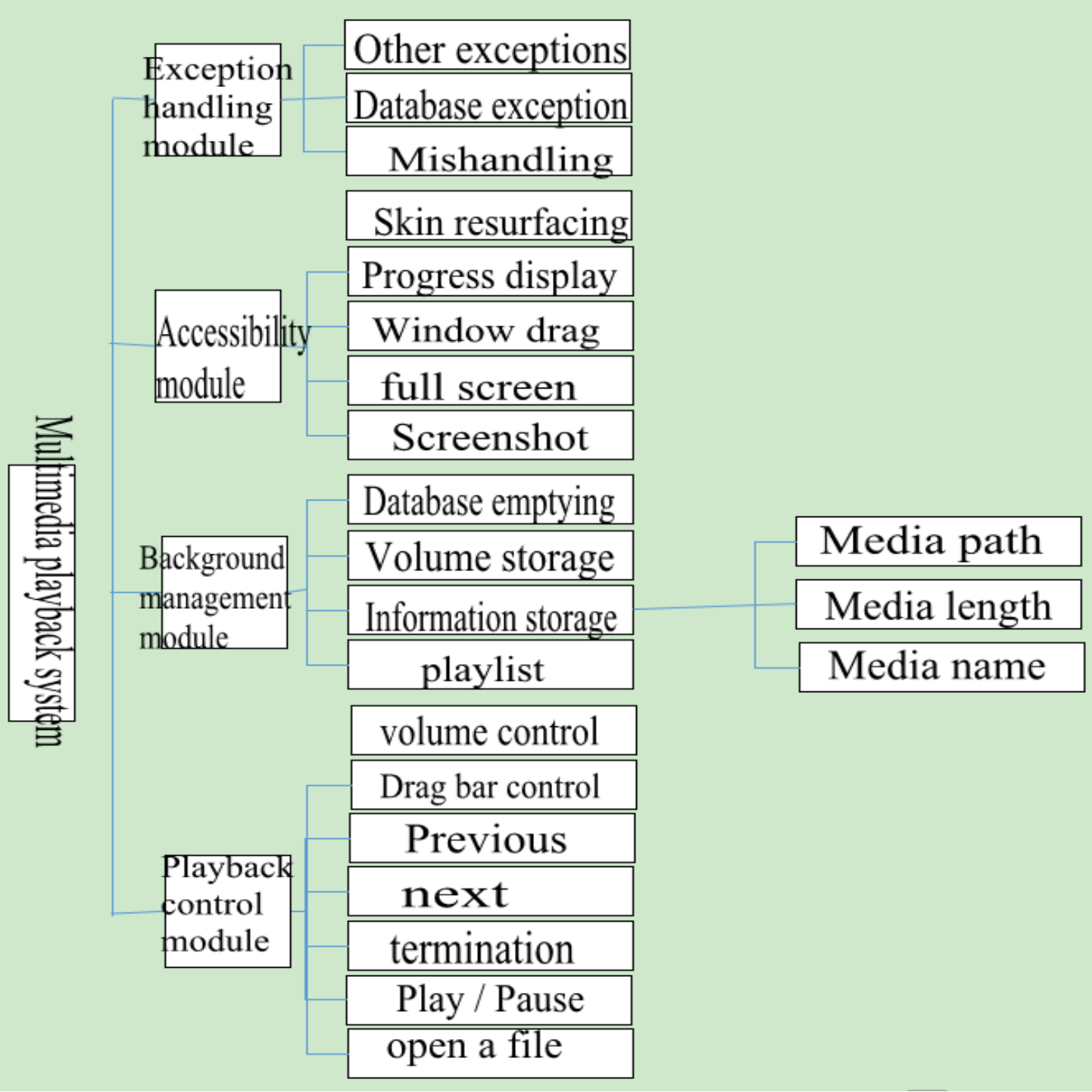

Figure 3.1 Functional structure of the multimedia playback system 
We use computer language programming technology in the processing of graphics and images. In the multimedia data processing, the editing of various media is fully integrated using MCI interface. When the main program chooses to load the background music, the background music is played, and the background music is paused when the pause is selected. At the same time, the user control objects of Visual Basic and Visual $\mathrm{C}++$ are fully utilized to solve the problem of possible limitation in multimedia presentation. The following are the main function implementation functions in the multimedia playback system structure.

(1) File support function boolis File Supported(const QS tring\&filename);

(2) The following is the core code to open the file function: Tmain Form. Action_Open File Execute(Sender: T Object), which has no return value.

(3) Get file information function const QS tring\& file Info ( ); file initialization function void fileInit( )

(4) Play function T Main Form.btn_play Click(Sender: T Object)

(5) In addition, there is the plugin initialization and registration function void pluginInit ( )

(6) The core code of the function table of the previous function implementation: <script type="text/Javascript" $>/ *$ Javascript

(7) read the audio sampling function bool audio Read Samples (short* output, int channels, long samples, long \& samples Read, int); audio and video synchronization function definition: int Sync (File * fp, int auIndex, struct timeval * vtime);

(8) The core code of the next file playback function: void C My Player D $\lg ::$ On Open()

(9) Find function bool seek(long pos)

(10) The implementation of the progress drag bar is to use the track bar Change (Sender: T Object) function, which has no return value.

(11) Clear the video data function flush Video Packets() and clear the audio data function flush Audio Packets()

(12) Volume adjustment is an important function in the media playback process, because different users have different requirements for the sound level, so the volume is also stored in the database. The volume adjustment bar updates the volume value in the database to change the playback volume of the media player.

(13) Get the function of the next packet Media Packet*get Another Packet(int stream), etc.

(14) The function to achieve mute and restore volume is btn_mute Click(Sender: T Object), which has no return value.

(15) The timer function implementation uses the Timer1Timer (Sender: T Object) function and has no return value.

(16) The core code of the function that implements the stop function: bt_stop Click(Sender: $T$ Object)

Through the above media decoding, playback control, auxiliary playback function, interface and abnormal processing four application architectures can ensure the security and stability of the multimedia playback system, fast response and easy to maintain multimedia playback system. Improving the response speed to the multimedia playback system client is through load balancing and buffering data capabilities.

\section{The Overall Structural Design}

\section{The Overall Structure of Multimedia Playback System Based on Computer Network and New Media Technology}

The multimedia playback system based on computer network and new media technology mainly consists of three parts, including production, storage and network broadcast. The material is transferred by editing, recording, and reviewing. The documents in the production process are stored in the material library, and the finished products are put into the media library. The finished products need to be reviewed before they can enter the library to be broadcasted, and finally broadcasted to the broadcast server according to the standard of the program list. System structure is showing as follows. 


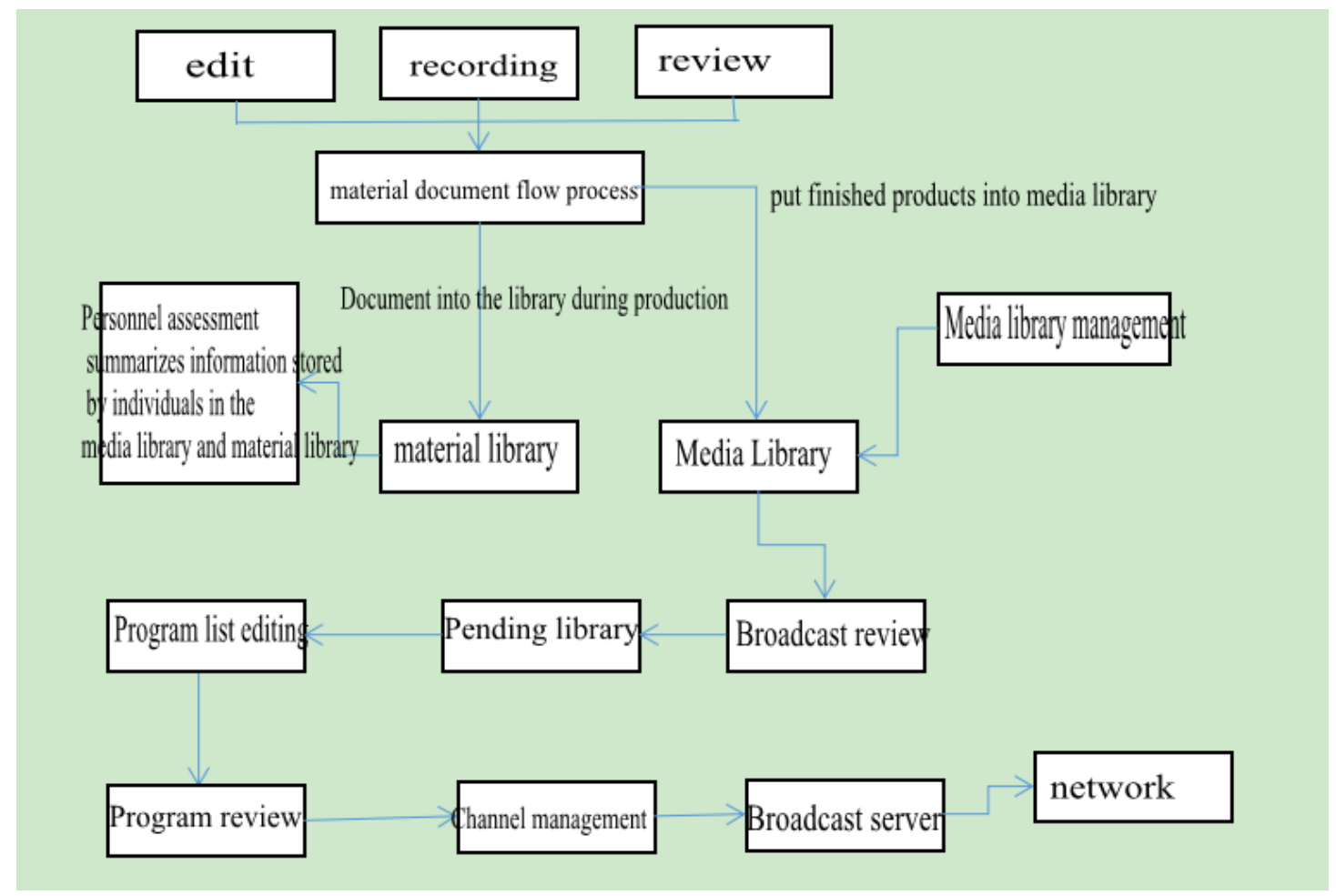

Figure 4.1 Overall System Architecture

\section{Implementation of Multimedia Playback System Based on Computer Network and New Media Technology}

Put Data_Into SQL Server

The information of the opened media file is written into the database, namely, the media information and personal setting data such as the name, length and path of the media file are written into the database to ensure that the user can memorize through the data to complete the previously unfinished playback of the media file.

If you want to do more with less in multimedia playback system design, you need to be good at using multimedia integration tools. In addition to the rich programming experience, the design and development of the multimedia playback system must fully understand the characteristics of multimedia, so it is different from the usual application system development. The preparation of the material takes up a considerable amount of work in the completion of the multimedia playback system with high technical and artistic content. It is the purpose of the multimedia playback system to facilitate the user to use multimedia efficiently. Therefore, the technology for developing a multimedia playback system has become more and more intensive with the development of computer networks and multimedia technologies.

\section{Conclusion}

In short, the development of computer networks and new media technologies directly determines the development of multimedia playback systems. As far as multimedia playback systems are concerned, advances in computer networks and new media technologies can not only break through the reality of current multimedia playback systems, but also broaden the reach of multimedia playback systems. It creates new technical and space support for the multimedia playback system based on the new media of computer network, which greatly enriches the carrier of multimedia playback system. The rapid development of computer networks and new media technologies has greatly promoted the progress of multimedia playback systems, which is in line with the current development trend of society, and even promotes in the future development. 


\section{Reference}

[1] X. L. Teng. Analysis of the Problems and Countermeasures in Online Advertising [A]. Wuhan University. Proceedings of the Conference on Web Based Business Management [C]. Wuhan University, 2010: 4.

[2] Eggers S J, Emer J S, Leby $\mathrm{H}$ M, et al. Simultaneous multithreading: A platform for next-generation processors[J]. Micro, IEEE, 1997,17(5): 12-19.

[3] Y. L. Wang, J. Q. Xian and J. Bai. P2P-based Streaming Media Technology [J]. Computer Applications, 2005, 06: 1267-1270.

[4] L. L. Zhang. Visual C++ Audio/Video Technology Development and Practice. Tsinghua University Press.

[5] Z. Y. Zhang and W. Wang. Application Research of Java Technology in Multimedia System [J]. Computer and Information Technology, 2010 (06).

[6] F. F. Yang. Discussion on Media Technology and Social Development [J]. Theoretical Monthly, 2006, (6). doi: 10.396 9/j.issn. 1004-0544. 2006. 06. 024.

[7] H. Zhu. Discussion on the Influence of Network Technology Development on Computer and Information Technology [J]. Information and Computer (Theoretical Edition), 2011 (08): $112+114$.

[8] Y. Xing. The Development of Broadcasting New Media in the New Media Environment Taking the Innovation Practice of Dong Guang Xin Wentai as An Example [J]. Audiovisual, 2015 (02): 38-40.215.

[9] R. Liu. Research on the Influence of Computer Network Technology Development on Internet New Media [J]. Information and Computer, 2017, (19).

[10]Z. Z. Wei and C. H. Liu. Research on the Influence of Computer Network Technology on the Development of Internet Media [J]. Science and Technology Innovation and Application, 2016, (36): 86. 